\title{
G3(MP2) Ring Strain in Bicyclic Phosphorus Heterocycles and Their Hydrocarbon Analogues
}

\author{
Mark L.G. Borst, Andreas W. Ehlers and Koop Lammertsma* \\ Department of Organic and Inorganic Chemistry, Faculty of Sciences, Vrije Universiteit \\ De Boelelaan 1083, NL-1081 HV, Amsterdam, The Netherlands \\ * Corresponding author: tel.: +31-20-598-7474 \\ E-mail address: k.lammertsma@few.vu.nl
}

Table of contents: Structures and energies

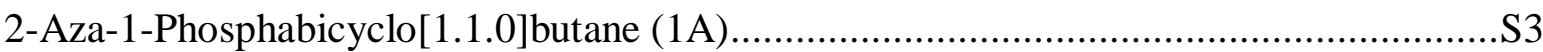

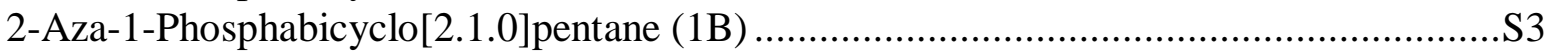

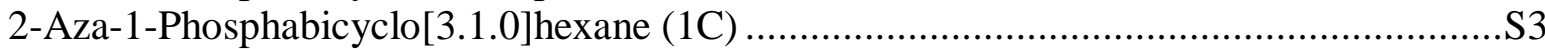

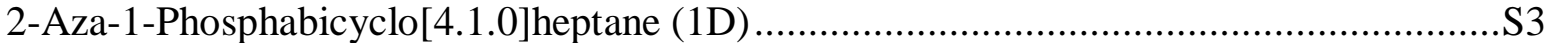

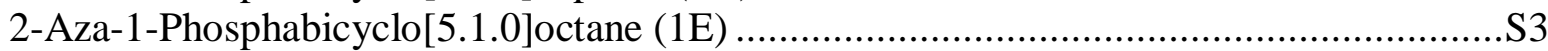

2-Aza-1-Phosphabicyclo[5.1.0]octane (1E) second conformer..................................S4

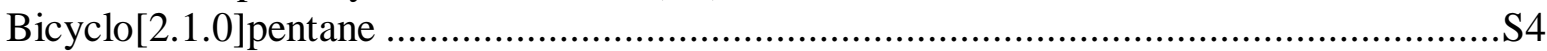

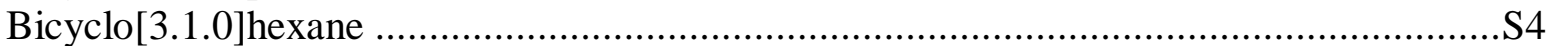

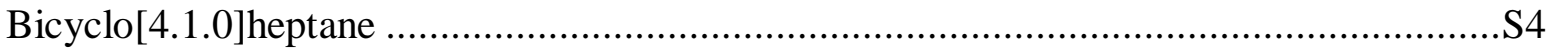

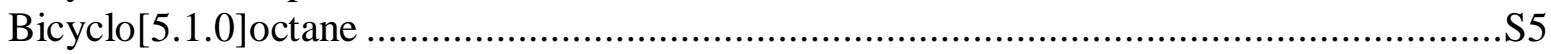

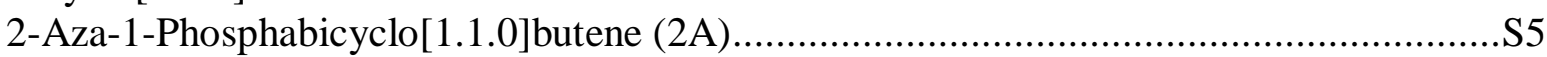

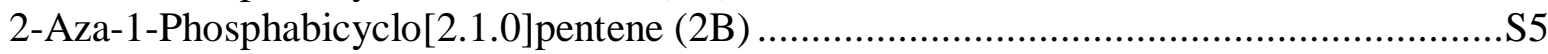

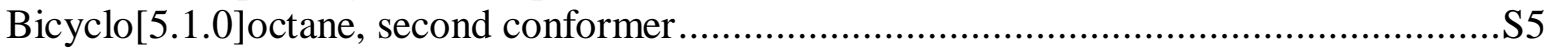

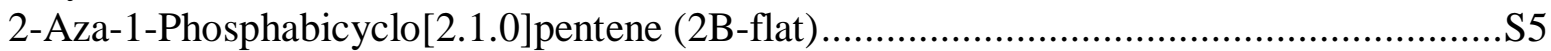

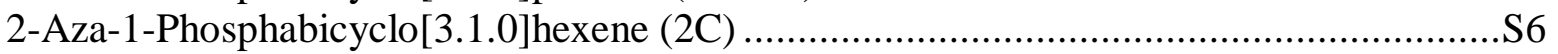

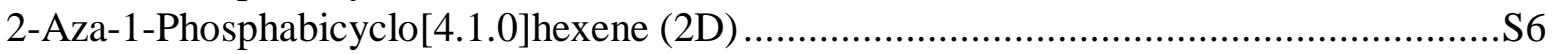

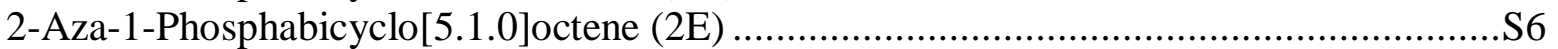

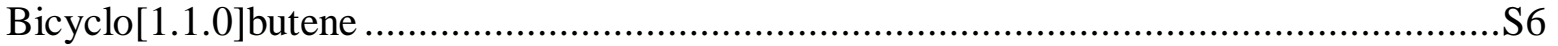

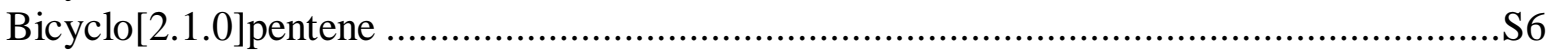

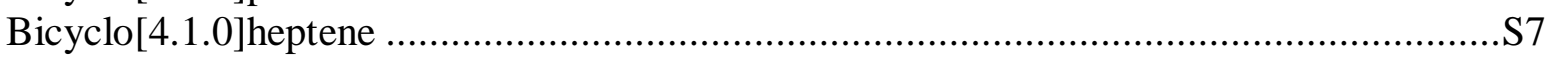

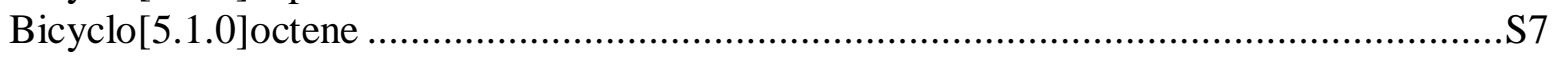

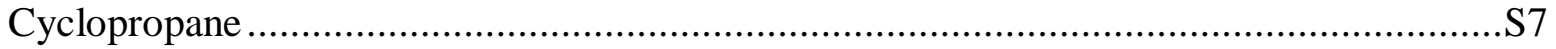

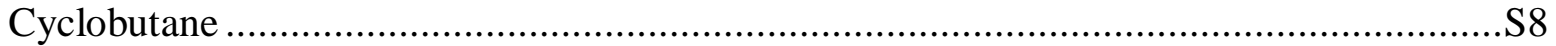




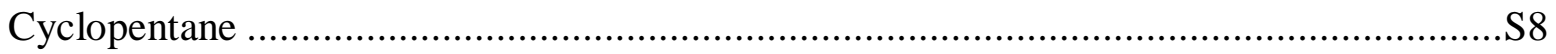

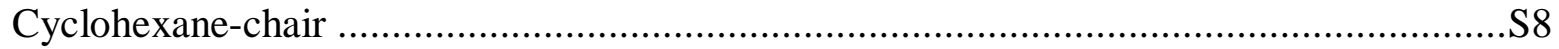

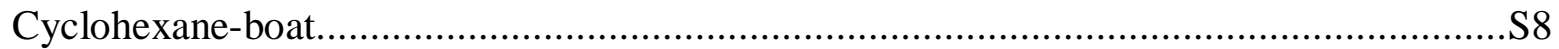

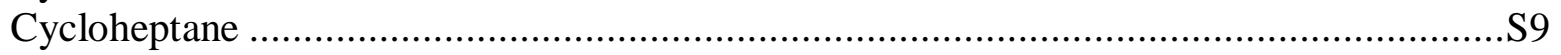

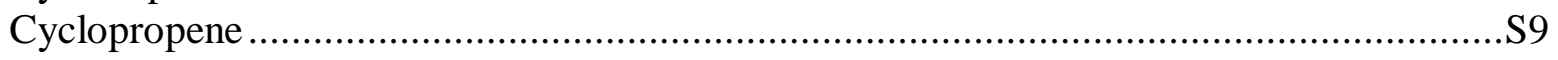

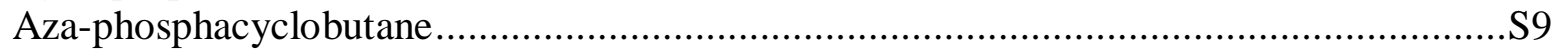

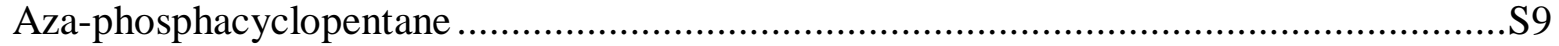

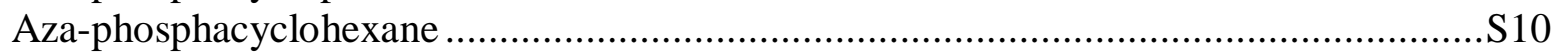

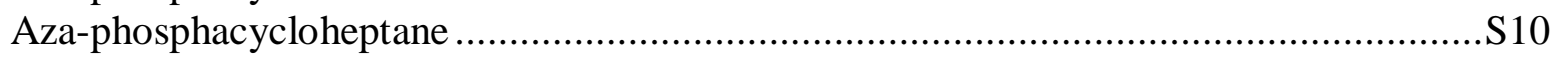

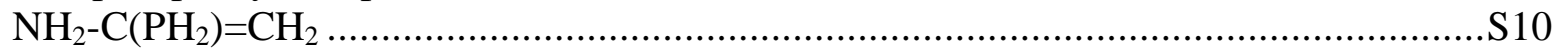

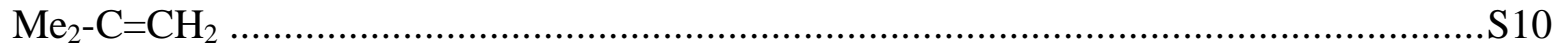

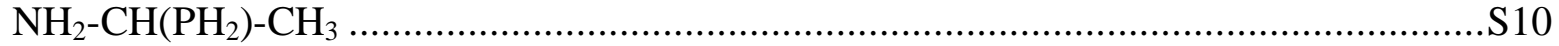

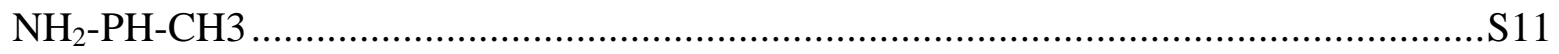

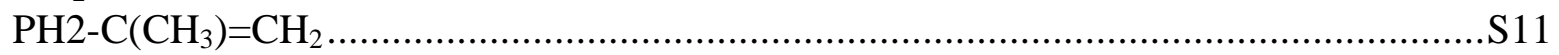

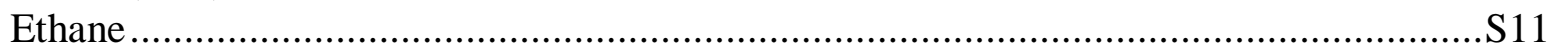

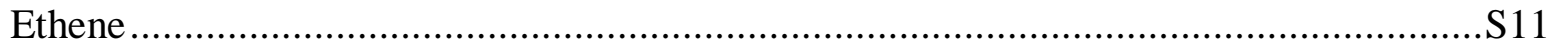


Computational Details.

All geometry optimizations were performed with the Gaussian 98 suite of programs, using the B3LYP exchangecorrelation potentials and 6-31G(*) basis-set (see mss). All structures were confirmed to have only positive force constants. The global minimum was subjected to a G3(MP2) evaluation of the energy. All values in $\mathrm{kcal} / \mathrm{mol}$.

$\begin{array}{lccc}\text { 2-Aza-1-Phosphabicyclo[1.1.0]butane (1A) } \\ \text { H } & -2.141 & -0.245 & 0.275 \\ \mathrm{~N} & 1.041 & -0.612 & -0.440 \\ \mathrm{C} & -0.029 & -0.812 & 0.506 \\ \mathrm{P} & 0.103 & 0.948 & 0.074 \\ \mathrm{H} & 1.939 & -0.626 & 0.069 \\ \mathrm{H} & 0.042 & -1.297 & 1.473 \\ \mathrm{C} & -1.203 & -0.362 & -0.265 \\ \mathrm{H} & -1.281 & -0.728 & -1.288\end{array}$

$\begin{array}{lrrr}\text { 2-Aza-1-Phosphabicyclo[4.1.0]heptane (1D) } \\ \mathrm{H} & 0.850 & 2.478 & 0.081 \\ \mathrm{C} & 0.768 & 1.477 & -0.362 \\ \mathrm{C} & 1.524 & -0.918 & -0.121 \\ \mathrm{P} & -1.226 & -0.708 & -0.361 \\ \mathrm{~N} & 0.221 & -1.444 & 0.289 \\ \mathrm{C} & -0.691 & 1.072 & -0.392 \\ \mathrm{C} & 1.687 & 0.508 & 0.379 \\ \mathrm{H} & 1.678 & -0.935 & -1.214 \\ \mathrm{H} & -2.437 & 1.095 & 0.986 \\ \mathrm{C} & -1.437 & 0.690 & 0.846 \\ \mathrm{H} & -0.870 & 0.562 & 1.764 \\ \mathrm{H} & 1.484 & 0.521 & 1.455 \\ \mathrm{H} & 1.121 & 1.560 & -1.398 \\ \mathrm{H} & 2.293 & -1.559 & 0.325 \\ \mathrm{H} & 2.726 & 0.831 & 0.242 \\ \mathrm{H} & 0.171 & -2.451 & 0.149 \\ \mathrm{H} & -1.278 & 1.657 & -1.100\end{array}$

$\mathrm{E}\left(\mathrm{QCISD} / 6-31 \mathrm{G}^{*}\right)=-297271.32$

G3(MP2) Enthalpy=-297439.30

2-Aza-1-Phosphabicyclo[2.1.0]pentane (1B)

$\begin{array}{lrrr}\mathrm{H} & -1.623 & 1.261 & 0.779 \\ \mathrm{C} & -1.279 & 0.641 & -0.061 \\ \mathrm{~N} & -1.070 & -0.790 & 0.282 \\ \mathrm{C} & 0.139 & 0.955 & -0.504 \\ \mathrm{P} & 0.614 & -0.854 & -0.265 \\ \mathrm{H} & 0.800 & 0.880 & 1.625 \\ \mathrm{H} & 2.136 & 1.028 & 0.386 \\ \mathrm{H} & -2.006 & 0.723 & -0.874 \\ \mathrm{H} & 0.376 & 1.592 & -1.351 \\ \mathrm{C} & 1.113 & 0.718 & 0.594 \\ \mathrm{H} & -1.242 & -1.031 & 1.259\end{array}$

$\mathrm{E}(\mathrm{QCISD} / 6-31 \mathrm{G} *)=-321869.43$

G3(MP2) Enthalpy=-322071.00

2-Aza-1-Phosphabicyclo[3.1.0]hexane (1C)

$\begin{array}{lrrr}\mathrm{H} & -0.581 & 0.711 & 1.787 \\ \mathrm{C} & -0.417 & 1.037 & -0.419 \\ \mathrm{P} & -0.997 & -0.714 & -0.331 \\ \mathrm{~N} & 0.627 & -1.268 & -0.030 \\ \mathrm{C} & 1.538 & -0.199 & 0.390 \\ \mathrm{C} & 1.099 & 1.046 & -0.382 \\ \mathrm{H} & -0.894 & 1.717 & -1.121 \\ \mathrm{H} & 1.492 & 1.954 & 0.092 \\ \mathrm{C} & -1.143 & 0.721 & 0.856 \\ \mathrm{H} & 1.485 & 1.002 & -1.405 \\ \mathrm{H} & 0.681 & -2.095 & 0.561 \\ \mathrm{H} & 1.487 & -0.004 & 1.471 \\ \mathrm{H} & 2.572 & -0.474 & 0.157 \\ \mathrm{H} & -2.146 & 1.130 & 0.962\end{array}$

$\mathrm{E}\left(\mathrm{QCISD} / 6-31 \mathrm{G}^{*}\right)=-371061.78$

G3(MP2) Enthalpy=-371326.33

$\begin{array}{lccr}\text { 2-Aza-1-Phosphabicyclo[5.1.0] } 0 \text { ctane } & \text { (1E) } \\ \mathrm{H} & 0.667 & 1.476 & -1.644 \\ \mathrm{C} & 0.493 & 1.639 & -0.573 \\ \mathrm{H} & 0.535 & 2.726 & -0.419 \\ \mathrm{C} & -0.921 & 1.201 & -0.228 \\ \mathrm{H} & 2.539 & 1.631 & -0.012 \\ \mathrm{P} & -1.589 & -0.508 & -0.309 \\ \mathrm{H} & -0.482 & 0.395 & 1.798 \\ \mathrm{H} & -2.166 & 1.039 & 1.634 \\ \mathrm{H} & -1.659 & 1.904 & -0.619 \\ \mathrm{C} & -1.296 & 0.635 & 1.121 \\ \mathrm{~N} & -0.206 & -1.542 & -0.424 \\ \mathrm{C} & 1.002 & -1.477 & 0.397 \\ \mathrm{H} & 1.478 & -1508611 & 0.290 \\ \mathrm{H} & -0.545 & -2.498 & -0.497 \\ \mathrm{H} & 0.783 & -1.317 & 1.465 \\ \mathrm{H} & 1.481 & -2.460 & 0.323 \\ \mathrm{C} & 1.996 & -0.429 & -0.088 \\ \mathrm{C} & 1.651 & 1.027 & 0.214 \\ \mathrm{H} & 2.131 & -0.563 & -1.167 \\ \mathrm{H} & 2.962 & -0.649 & 0.387 \\ \mathrm{E}\left(\mathrm{QCISD} / 6-31 \mathrm{G}^{*}\right)=-395644.61 & \\ \mathrm{G} 3(\mathrm{MP} 2) & \text { Enthalpy=-395942.49 } & \\ & & & \end{array}$




\begin{tabular}{lrrr}
\multicolumn{4}{l}{ 2-Aza-1-Phosphabicyclo[5.1.0]octane (1E) } \\
second conformer \\
H & 0.792 & 1.496 & -1.532 \\
$\mathrm{C}$ & 0.376 & 1.602 & -0.521 \\
$\mathrm{H}$ & 0.223 & 2.680 & -0.371 \\
$\mathrm{C}$ & -1.013 & 0.978 & -0.492 \\
$\mathrm{H}$ & 2.129 & 1.912 & 0.675 \\
$\mathrm{P}$ & -1.438 & -0.821 & -0.262 \\
$\mathrm{H}$ & -1.199 & 0.715 & 1.702 \\
$\mathrm{H}$ & -2.784 & 1.044 & 0.853 \\
$\mathrm{H}$ & -1.647 & 1.395 & -1.276 \\
$\mathrm{C}$ & -1.755 & 0.702 & 0.769 \\
$\mathrm{~N}$ & 0.052 & -1.380 & 0.460 \\
$\mathrm{C}$ & 1.247 & -1.271 & -0.387 \\
$\mathrm{H}$ & 0.904 & 0.942 & 1.465 \\
$\mathrm{H}$ & -0.068 & -2.321 & 0.825 \\
$\mathrm{H}$ & 1.793 & -2.222 & -0.386 \\
$\mathrm{H}$ & 0.967 & -1.078 & -1.432 \\
$\mathrm{C}$ & 2.159 & -0.151 & 0.108 \\
$\mathrm{C}$ & 1.399 & 1.112 & 0.505 \\
$\mathrm{H}$ & 2.889 & 0.082 & -0.679 \\
$\mathrm{H}$ & 2.730 & -0.502 & 0.976
\end{tabular}

$\mathrm{E}\left(\mathrm{QCISD} / 6-31 \mathrm{G}^{*}\right)=-395644.94$

G3(MP2) Enthalpy= -395941.11

$\begin{array}{lrrr}\text { Bicyclo[2.1.0]pentane } & & \\ \mathrm{H} & -0.609 & 1.459 & 1.278 \\ \mathrm{C} & 0.246 & 0.988 & 0.779 \\ \mathrm{C} & 0.246 & 0.988 & -0.779 \\ \mathrm{C} & 0.246 & -0.538 & 0.763 \\ \mathrm{C} & 0.246 & -0.538 & -0.763 \\ \mathrm{H} & -1.870 & -0.463 & 0.000 \\ \mathrm{H} & -1.081 & -2.112 & 0.000 \\ \mathrm{H} & 1.166 & 1.413 & 1.187 \\ \mathrm{H} & 0.794 & -1.181 & 1.444 \\ \mathrm{C} & -0.943 & -1.035 & 0.000 \\ \mathrm{H} & 1.166 & 1.413 & -1.187 \\ \mathrm{H} & -0.609 & 1.459 & -1.278 \\ \mathrm{H} & 0.794 & -1.181 & -1.444\end{array}$

\section{Bicyclo[3.1.0]hexane}

$\begin{array}{lrrr}\mathrm{C} & -0.916 & -1.292 & 0.000 \\ \mathrm{C} & 0.292 & -0.809 & 0.754 \\ \mathrm{C} & 0.292 & -0.809 & -0.754 \\ \mathrm{C} & 0.292 & 0.633 & -1.220 \\ \mathrm{C} & -0.197 & 1.432 & 0.000 \\ \mathrm{C} & 0.292 & 0.633 & 1.220 \\ \mathrm{H} & 0.874 & -1.540 & 1.309 \\ \mathrm{H} & -1.820 & -0.689 & 0.000 \\ \mathrm{H} & 0.874 & -1.540 & -1.309 \\ \mathrm{H} & 1.317 & 0.925 & -1.479 \\ \mathrm{H} & -0.332 & 0.803 & -2.105 \\ \mathrm{H} & -1.289 & 1.488 & 0.000 \\ \mathrm{H} & 0.171 & 2.462 & 0.000 \\ \mathrm{H} & -0.332 & 0.803 & 2.105 \\ \mathrm{H} & 1.317 & 0.925 & 1.479 \\ \mathrm{H} & -1.103 & -2.361 & 0.000\end{array}$

\section{$\mathrm{E}\left(\mathrm{QCISD} / 6-31 \mathrm{G}^{*}\right)=-146754.68$}

G3(MP2) Enthalpy=-146943.73

$\begin{array}{lrrr}\text { Bicyclo[4.1.0]heptane } & & \\ \mathrm{H} & -0.469 & 2.408 & -0.614 \\ \mathrm{C} & -0.346 & 1.514 & 0.007 \\ \mathrm{C} & -1.317 & -0.760 & 0.478 \\ \mathrm{C} & 1.032 & -0.675 & -0.470 \\ \mathrm{C} & -0.215 & -1.508 & -0.272 \\ \mathrm{C} & 0.966 & 0.831 & -0.353 \\ \mathrm{C} & -1.560 & 0.600 & -0.166 \\ \mathrm{H} & -1.045 & -0.625 & 1.533 \\ \mathrm{H} & 1.224 & -0.065 & 1.662 \\ \mathrm{H} & -0.607 & -1.788 & -1.259 \\ \mathrm{H} & 1.603 & 1.383 & -1.040 \\ \mathrm{H} & -1.758 & 0.453 & -1.236 \\ \mathrm{H} & -0.308 & 1.866 & 1.046 \\ \mathrm{H} & -2.234 & -1.361 & 0.470 \\ \mathrm{H} & 1.710 & -1.059 & -1.231 \\ \mathrm{H} & 0.036 & -2.444 & 0.243 \\ \mathrm{C} & 1.693 & 0.015 & 0.685 \\ \mathrm{H} & -2.449 & 1.081 & 0.259 \\ \mathrm{H} & 2.777 & 0.051 & 0.717\end{array}$

$\mathrm{E}(\mathrm{QCISD} / 6-31 \mathrm{G} *)=-122142.55$

G3(MP2) Enthalpy=-122300.84
$\mathrm{E}(\mathrm{QCISD} / 6-31 \mathrm{G} *)=-171342.72$

G3(MP2) Enthalpy=-171563.25 


\section{Bicyclo[5.1.0]octane}

$\begin{array}{lrrr}\mathrm{H} & 1.414 & 1.126 & 1.349 \\ \mathrm{C} & 0.326 & 1.264 & 1.305 \\ \mathrm{C} & 0.326 & 1.264 & -1.305 \\ \mathrm{C} & 0.326 & -1.201 & -0.752 \\ \mathrm{C} & -0.361 & -0.090 & -1.510 \\ \mathrm{C} & -0.361 & -0.090 & 1.510 \\ \mathrm{C} & -0.051 & 1.969 & 0.000 \\ \mathrm{H} & 0.066 & 1.933 & -2.135 \\ \mathrm{H} & 1.249 & -1.544 & -1.216 \\ \mathrm{H} & -0.373 & -0.347 & -2.577 \\ \mathrm{H} & -1.413 & -0.015 & 1.207 \\ \mathrm{H} & 0.411 & 2.965 & 0.000 \\ \mathrm{H} & 0.066 & 1.933 & 2.135 \\ \mathrm{H} & 1.414 & 1.126 & -1.349 \\ \mathrm{H} & 1.249 & -1.544 & 1.216 \\ \mathrm{H} & -1.413 & -0.015 & -1.207 \\ \mathrm{C} & 0.326 & -1.201 & 0.752 \\ \mathrm{H} & -1.137 & 2.140 & 0.000 \\ \mathrm{C} & -0.453 & -2.247 & 0.000 \\ \mathrm{H} & -1.536 & -2.154 & 0.000 \\ \mathrm{H} & -0.373 & -0.347 & 2.577 \\ \mathrm{H} & -0.095 & -3.272 & 0.000\end{array}$

$\mathrm{E}\left(\mathrm{QCISD} / 6-31 \mathrm{G}^{*}\right)=-195929.75$

G3(MP2) Enthalpy=-196182.78

\section{2-Aza-1-Phosphabicyclo[1.1.0]butene (2A)}

$\begin{array}{lrrr}\mathrm{C} & 0.403 & -1.017 & 0.518 \\ \mathrm{~N} & -0.065 & 1.141 & 0.393 \\ \mathrm{C} & -0.371 & -0.033 & 1.132 \\ \mathrm{P} & 0.031 & -0.097 & -0.916 \\ \mathrm{H} & -0.849 & 1.806 & 0.342 \\ \mathrm{H} & 0.656 & -2.041 & 0.749 \\ \mathrm{E}\left(\mathrm{QCISD} / 6-31 \mathrm{G}^{*}\right)=-296487.32 & \\ \text { G3(MP2) Enthalpy=-296651.58 } & \end{array}$

2-Aza-1-Phosphabicyclo[2.1.0]pentene (2B)

$\begin{array}{lrrr}\mathrm{C} & 0.049 & 0.878 & -0.540 \\ \mathrm{C} & 1.011 & 1.066 & 0.368 \\ \mathrm{P} & 0.801 & -0.709 & -0.192 \\ \mathrm{H} & 1.022 & 1.579 & 1.328 \\ \mathrm{C} & -1.363 & 0.472 & -0.181 \\ \mathrm{H} & -2.009 & 0.192 & -1.021 \\ \mathrm{H} & -1.873 & 1.196 & 0.462 \\ \mathrm{~N} & -0.848 & -0.748 & 0.548 \\ \mathrm{H} & -1.397 & -1.597 & 0.387\end{array}$

$\mathrm{E}(\mathrm{QCISD} / 6-31 \mathrm{G} *)=-321069.07$

G3(MP2) Enthalpy=-321267.84

\section{Bicyclo[5.1.0] octane, second conformer}

$\begin{array}{lrrr}\mathrm{H} & 0.271 & 1.839 & 2.124 \\ \mathrm{C} & -0.030 & 1.188 & 1.293 \\ \mathrm{C} & -0.030 & 1.188 & -1.293 \\ \mathrm{C} & -0.030 & -1.361 & -0.752 \\ \mathrm{C} & 0.452 & -0.222 & -1.625 \\ \mathrm{C} & 0.452 & -0.222 & 1.625 \\ \mathrm{C} & 0.520 & 1.786 & 0.000 \\ \mathrm{H} & -1.126 & 1.216 & -1.275 \\ \mathrm{H} & 0.218 & -2.330 & -1.183 \\ \mathrm{H} & 1.551 & -0.223 & -1.631 \\ \mathrm{H} & 0.146 & -0.448 & 2.655 \\ \mathrm{H} & 1.615 & 1.697 & 0.000 \\ \mathrm{H} & -1.126 & 1.216 & 1.275 \\ \mathrm{H} & 0.271 & 1.839 & -2.124 \\ \mathrm{H} & 0.218 & -2.330 & 1.183 \\ \mathrm{H} & 0.146 & -0.448 & -2.655 \\ \mathrm{C} & -0.030 & -1.361 & 0.752 \\ \mathrm{H} & 0.300 & 2.861 & 0.000 \\ \mathrm{C} & -1.335 & -1.336 & 0.000 \\ \mathrm{H} & -1.900 & -0.411 & 0.000 \\ \mathrm{H} & 1.551 & -0.223 & 1.631 \\ \mathrm{H} & -1.956 & -2.227 & 0.000\end{array}$

$\mathrm{E}\left(\mathrm{QCISD} / 6-31 \mathrm{G}^{*}\right)=-195928.12$

G3(MP2) Enthalpy=-196180.93

$\begin{array}{lrrc}\text { 2-Aza-1-Phosphabicyclo[2.1.0]pentene } & \text { (2B-flat) } \\ \text { H } & -1.955 & -0.727 & 0.885 \\ \text { C } & -1.382 & -0.419 & 0.000 \\ \text { N } & -0.084 & -1.149 & 0.000 \\ \text { C } & -1.186 & 1.044 & 0.000 \\ \text { P } & 1.211 & -0.137 & 0.000 \\ \text { C } & 0.187 & 1.284 & 0.000 \\ \text { H } & 0.636 & 2.274 & 0.000 \\ \text { H } & -1.955 & -0.728 & -0.885 \\ \text { H } & -0.014 & -2.164 & 0.000\end{array}$

$\mathrm{E}\left(\mathrm{QCISD} / 6-31 \mathrm{G}^{*}\right)=-321091.51$

G3(MP2) Enthalpy=-321291.55 


$\begin{array}{lrrr}\text { 2-Aza-1-Phosphabicyclo[3.1.0]hexene } & (\mathbf{2 C}) \\ \mathrm{H} & 1.511 & 0.800 & -1.499 \\ \mathrm{~N} & 0.365 & -1.297 & 0.141 \\ \mathrm{P} & -1.131 & -0.522 & -0.344 \\ \mathrm{C} & -0.237 & 1.034 & -0.285 \\ \mathrm{C} & 1.234 & 0.934 & -0.450 \\ \mathrm{C} & 1.489 & -0.373 & 0.360 \\ \mathrm{H} & 0.339 & -2.078 & 0.792 \\ \mathrm{H} & 2.424 & -0.838 & 0.029 \\ \mathrm{C} & -1.148 & 1.048 & 0.676 \\ \mathrm{H} & 1.595 & -0.100 & 1.420 \\ \mathrm{H} & -1.285 & 1.504 & 1.653 \\ \mathrm{H} & 1.803 & 1.767 & -0.026\end{array}$

$\mathrm{E}\left(\mathrm{QCISD} / 6-31 \mathrm{G}^{*}\right)=-345689.19$

G3(MP2) Enthalpy=-345920.29

$\begin{array}{lcrr}\text { 2-Aza-1-Phosphabicyclo[4.1.0]hexene } & (\mathbf{2 D}) \\ \mathrm{C} & 1.378 & -1.082 & 0.678 \\ \mathrm{P} & 1.361 & 0.409 & -0.402 \\ \mathrm{C} & -1.217 & 1.167 & -0.069 \\ \mathrm{C} & -1.038 & -1.386 & -0.399 \\ \mathrm{C} & -1.751 & -0.220 & 0.318 \\ \mathrm{C} & 0.403 & -1.106 & -0.204 \\ \mathrm{~N} & 0.158 & 1.409 & 0.404 \\ \mathrm{H} & -1.866 & 1.921 & 0.389 \\ \mathrm{H} & -1.336 & -2.347 & 0.032 \\ \mathrm{H} & -2.824 & -0.255 & 0.095 \\ \mathrm{H} & 1.707 & -1.513 & 1.615 \\ \mathrm{H} & 0.383 & 2.402 & 0.351 \\ \mathrm{H} & -1.627 & -0.345 & 1.399 \\ \mathrm{H} & -1.314 & 1.282 & -1.163 \\ \mathrm{H} & -1.294 & -1.389 & -1.465\end{array}$

$\mathrm{E}\left(\mathrm{QCISD} / 6-31 \mathrm{G}^{*}\right)=-370288.86$

G3(MP2) Enthalpy=-370551.33
2-Aza-1-Phosphabicyclo[5.1.0]octene (2E)

$\begin{array}{lrrr}\mathrm{H} & -0.335 & 1.162 & 1.539 \\ \mathrm{C} & -0.377 & 1.587 & 0.523 \\ \mathrm{H} & -1.416 & 1.099 & -1.296 \\ \mathrm{~N} & 0.881 & 1.434 & -0.206 \\ \mathrm{H} & -0.532 & 2.665 & 0.642 \\ \mathrm{P} & 1.657 & -0.096 & -0.438 \\ \mathrm{H} & -3.022 & -0.610 & -0.232 \\ \mathrm{H} & 1.534 & 2.197 & -0.040 \\ \mathrm{H} & -2.044 & -0.526 & 1.229 \\ \mathrm{C} & 1.179 & -1.190 & 0.959 \\ \mathrm{C} & 0.305 & -1.209 & -0.035 \\ \mathrm{H} & -2.456 & 1.646 & 0.012 \\ \mathrm{H} & 1.305 & -1.530 & 1.981 \\ \mathrm{C} & -1.088 & -1.532 & -0.417 \\ \mathrm{H} & -1.390 & -2.505 & -0.014 \\ \mathrm{H} & -1.178 & -1.576 & -1.508 \\ \mathrm{C} & -2.005 & -0.430 & 0.137 \\ \mathrm{C} & -1.596 & 1.005 & -0.218\end{array}$

$\mathrm{E}\left(\mathrm{QCISD} / 6-31 \mathrm{G}^{*}\right)=-394881.30$

G3(MP2) Enthalpy=-395176.69

\section{Bicyclo[1.1.0]butene}

$\begin{array}{lrrr}\mathrm{H} & 1.3453 & -0.4029 & 1.1479 \\ \mathrm{C} & 1.0914 & -0.0877 & 0.1417 \\ \mathrm{C} & -0.1578 & -0.8891 & -0.3553 \\ \mathrm{C} & -0.0206 & 0.8569 & 0.0049 \\ \mathrm{H} & 1.9572 & 0.0080 & -0.5130 \\ \mathrm{H} & -2.1703 & -0.0383 & 0.3082 \\ \mathrm{C} & -1.0989 & -0.0739 & 0.1852 \\ \mathrm{H} & -0.0166 & 1.5966 & -0.8019 \\ \mathrm{E}\left(\mathrm{QCISD} / 6-31 \mathrm{G}^{*}\right)=-96756.96 & \\ \text { G3(MP2) Enthalpy=-96881.69 } & \end{array}$

\section{Bicyclo[2.1.0]pentene}

$\begin{array}{lrrr}\mathrm{H} & 1.414 & -1.455 & 0.720 \\ \mathrm{C} & 1.062 & -0.756 & -0.046 \\ \mathrm{C} & 0.855 & 0.784 & 0.328 \\ \mathrm{C} & -0.403 & -0.734 & -0.362 \\ \mathrm{C} & -0.518 & 0.763 & -0.374 \\ \mathrm{H} & -2.024 & -0.392 & 1.185 \\ \mathrm{H} & -0.770 & 1.366 & -1.241 \\ \mathrm{H} & 1.694 & -0.835 & -0.935 \\ \mathrm{H} & 0.799 & 0.920 & 1.416 \\ \mathrm{C} & -1.449 & -0.234 & 0.277 \\ \mathrm{H} & 1.608 & 1.462 & -0.086\end{array}$

$\mathrm{E}\left(\mathrm{QCISD} / 6-31 \mathrm{G}^{*}\right)=-121330.94$

G3(MP2) Enthalpy=-121487.95 


\section{Bicyclo[3.1.0]hexene}

$\begin{array}{lrrr}\mathrm{C} & 1.578 & -0.346 & 0.489 \\ \mathrm{C} & 0.921 & 0.626 & -0.444 \\ \mathrm{C} & 0.619 & -0.835 & -0.269 \\ \mathrm{C} & -0.820 & -1.168 & -0.268 \\ \mathrm{C} & -1.338 & 0.156 & 0.387 \\ \mathrm{C} & -0.378 & 1.316 & -0.006 \\ \mathrm{H} & 1.436 & 0.998 & -1.329 \\ \mathrm{H} & 2.112 & -0.450 & 1.426 \\ \mathrm{H} & -0.779 & 1.880 & -0.855 \\ \mathrm{H} & -1.207 & -1.283 & -1.286 \\ \mathrm{H} & -1.096 & -2.050 & 0.318 \\ \mathrm{H} & -1.320 & 0.012 & 1.471 \\ \mathrm{H} & -2.372 & 0.367 & 0.096 \\ \mathrm{H} & -0.259 & 2.023 & 0.824\end{array}$

$\mathrm{E}\left(\mathrm{QCISD} / 6-31 \mathrm{G}^{*}\right)=-145957.63$

G3(MP2) Enthalpy=-146144.98

\section{Bicyclo[4.1.0]heptene}

C

C

C

C

C

$\mathrm{C}$

$\mathrm{H}$

$\mathrm{H}$

$\mathrm{H}$

$\mathrm{H}$

$\mathrm{H}$

$\mathrm{H}$

$\mathrm{H}$

$\mathrm{H}$

$\mathrm{H}$

$\begin{array}{rrr}1.890 & -0.367 & 0.412 \\ 0.833 & -0.825 & -0.207 \\ 0.061 & 1.517 & 0.172 \\ -1.465 & -0.544 & 0.377 \\ -1.314 & 0.899 & -0.135 \\ -0.460 & -1.528 & -0.265 \\ 1.133 & 0.625 & -0.437 \\ 0.199 & 1.585 & 1.260 \\ -1.310 & -0.555 & 1.463 \\ -1.463 & 0.907 & -1.223 \\ -0.742 & -1.716 & -1.308 \\ 1.541 & 0.940 & -1.401 \\ -0.452 & -2.487 & 0.263 \\ 0.111 & 2.538 & -0.222 \\ -2.487 & -0.897 & 0.195 \\ -2.115 & 1.513 & 0.296 \\ 2.643 & -0.494 & 1.177\end{array}$

\section{Bicyclo[5.1.0]octene}

$\begin{array}{lrrr}\mathrm{H} & 1.213 & 1.128 & -1.272 \\ \mathrm{C} & 1.322 & 1.084 & -0.180 \\ \mathrm{C} & -1.260 & 1.243 & -0.299 \\ \mathrm{C} & -0.425 & -1.212 & -0.085 \\ \mathrm{C} & -1.503 & -0.246 & -0.486 \\ \mathrm{C} & 1.822 & -0.307 & 0.231 \\ \mathrm{C} & 0.015 & 1.562 & 0.484 \\ \mathrm{H} & -2.121 & 1.718 & 0.193 \\ \mathrm{H} & 2.866 & -0.426 & -0.083 \\ \mathrm{H} & -2.131 & -0.495 & -1.347 \\ \mathrm{H} & 1.175 & -1.555 & -1.419 \\ \mathrm{H} & 0.065 & 2.650 & 0.609 \\ \mathrm{H} & 2.115 & 1.798 & 0.073 \\ \mathrm{H} & -1.181 & 1.709 & -1.291 \\ \mathrm{C} & 1.003 & -1.476 & -0.339 \\ \mathrm{C} & -1.487 & -1.205 & 0.677 \\ \mathrm{H} & 1.811 & -0.376 & 1.326 \\ \mathrm{H} & -0.054 & 1.145 & 1.497 \\ \mathrm{H} & 1.320 & -2.419 & 0.119 \\ \mathrm{H} & -1.996 & -1.542 & 1.569 \\ & & & \end{array}$

$\mathrm{E}\left(\mathrm{QCISD} / 6-31 \mathrm{G}^{*}\right)=-195149.86$

G3(MP2) Enthalpy=-195400.54

\section{Cyclopropane}

$\begin{array}{lrrr}\mathrm{H} & 0.000 & 1.456 & 0.910 \\ \mathrm{C} & 0.000 & 0.867 & 0.000 \\ \mathrm{C} & 0.751 & -0.433 & 0.000 \\ \mathrm{C} & -0.751 & -0.433 & 0.000 \\ \mathrm{H} & 0.000 & 1.456 & -0.910 \\ \mathrm{H} & 1.261 & -0.728 & 0.910 \\ \mathrm{H} & 1.261 & -0.728 & -0.910 \\ \mathrm{H} & -1.261 & -0.728 & 0.910 \\ \mathrm{H} & -1.261 & -0.728 & -0.910\end{array}$

$\mathrm{E}\left(\mathrm{QCISD} / 6-31 \mathrm{G}^{*}\right)=-73730.11$

G3(MP2) Enthalpy=-73828.41

$\mathrm{E}\left(\mathrm{QCISD} / 6-31 \mathrm{G}^{*}\right)=-170558.31$

G3(MP2) Enthalpy=-170777.20 


$\begin{array}{lrrr}\text { Cyclobutane } & & & \\ \mathrm{H} & 1.986 & 0.000 & 0.451 \\ \mathrm{C} & 1.071 & 0.000 & -0.147 \\ \mathrm{C} & 0.000 & 1.071 & 0.147 \\ \mathrm{C} & 0.000 & -1.071 & 0.147 \\ \mathrm{C} & -1.071 & 0.000 & -0.147 \\ \mathrm{H} & -1.986 & 0.000 & 0.451 \\ \mathrm{H} & -1.344 & 0.000 & -1.207 \\ \mathrm{H} & 1.344 & 0.000 & -1.207 \\ \mathrm{H} & 0.000 & -1.986 & -0.451 \\ \mathrm{H} & 0.000 & -1.344 & 1.207 \\ \mathrm{H} & 0.000 & 1.986 & -0.451 \\ \mathrm{H} & 0.000 & 1.344 & 1.207\end{array}$

\section{Cyclohexane-chair}

$\mathrm{E}\left(\mathrm{QCISD} / 6-31 \mathrm{G}^{*}\right)=-98319.95$

G3(MP2) Enthalpy=-98449.02

$\begin{array}{lrrr}\text { Cyclopentane } & & & \\ \mathrm{H} & 1.911 & 1.218 & 0.144 \\ \mathrm{C} & 1.063 & 0.656 & -0.259 \\ \mathrm{C} & 0.976 & -0.778 & 0.260 \\ \mathrm{C} & -0.438 & -1.201 & -0.147 \\ \mathrm{C} & -1.296 & 0.077 & 0.002 \\ \mathrm{C} & -0.293 & 1.246 & 0.143 \\ \mathrm{H} & 1.162 & 0.644 & -1.351 \\ \mathrm{H} & 1.072 & -0.778 & 1.353 \\ \mathrm{H} & 1.753 & -1.437 & -0.139 \\ \mathrm{H} & -0.428 & -1.527 & -1.193 \\ \mathrm{H} & -0.821 & -2.038 & 0.445 \\ \mathrm{H} & -1.950 & 0.016 & 0.877 \\ \mathrm{H} & -1.948 & 0.213 & -0.866 \\ \mathrm{H} & -0.246 & 1.577 & 1.186 \\ \mathrm{H} & -0.573 & 2.117 & -0.456\end{array}$

$\mathrm{E}(\mathrm{QCISD} / 6-31 \mathrm{G} *)=-122926.76$

G3(MP2) Enthalpy=-123088.15

$\begin{array}{lrrr}\mathrm{H} & -2.489 & 0.000 & -0.131 \\ \mathrm{C} & -1.455 & 0.000 & 0.233 \\ \mathrm{C} & 0.727 & -1.260 & 0.233 \\ \mathrm{C} & 0.727 & 1.260 & 0.233 \\ \mathrm{C} & 1.455 & 0.000 & -0.233 \\ \mathrm{C} & -0.727 & 1.260 & -0.233 \\ \mathrm{C} & -0.727 & -1.260 & -0.233 \\ \mathrm{H} & 0.754 & -1.306 & 1.330 \\ \mathrm{H} & 0.754 & 1.306 & 1.330 \\ \mathrm{H} & 1.508 & 0.000 & -1.330 \\ \mathrm{H} & -0.754 & 1.306 & -1.330 \\ \mathrm{H} & -0.754 & -1.306 & -1.330 \\ \mathrm{H} & -1.508 & 0.000 & 1.330 \\ \mathrm{H} & 1.245 & -2.156 & -0.131 \\ \mathrm{H} & 1.245 & 2.156 & -0.131 \\ \mathrm{H} & 2.489 & 0.000 & 0.131 \\ \mathrm{H} & -1.245 & 2.156 & 0.131 \\ \mathrm{H} & -1.245 & -2.156 & 0.131\end{array}$

$\mathrm{E}(\mathrm{QCISD} / 6-31 \mathrm{G} *)=-147520.39$

G3(MP2) Enthalpy=-147713.04

\section{Cyclohexane-boat}

$\begin{array}{lrrr}\mathrm{H} & -2.173 & 0.269 & 0.835 \\ \mathrm{C} & -1.516 & 0.000 & 0.000 \\ \mathrm{C} & 0.653 & 1.211 & 0.395 \\ \mathrm{C} & 0.653 & -1.211 & -0.395 \\ \mathrm{C} & 1.516 & 0.000 & 0.000 \\ \mathrm{C} & -0.653 & -1.211 & 0.395 \\ \mathrm{C} & -0.653 & 1.211 & -0.395 \\ \mathrm{H} & 1.209 & 2.141 & 0.235 \\ \mathrm{H} & -1.209 & -2.141 & 0.235 \\ \mathrm{H} & 2.173 & -0.269 & 0.835 \\ \mathrm{H} & -0.418 & -1.174 & 1.465 \\ \mathrm{H} & -1.210 & 2.141 & -0.235 \\ \mathrm{H} & -2.172 & -0.269 & -0.835 \\ \mathrm{H} & 0.418 & 1.174 & 1.465 \\ \mathrm{H} & 1.210 & -2.141 & -0.235 \\ \mathrm{H} & 2.172 & 0.269 & -0.835 \\ \mathrm{H} & 0.418 & -1.174 & -1.465 \\ \mathrm{H} & -0.418 & 1.174 & -1.465\end{array}$

$\mathrm{E}\left(\mathrm{QCISD} / 6-31 \mathrm{G}^{*}\right)=-147513.90$

G3(MP2) Enthalpy=-147706.78 


$\begin{array}{lrrr}\text { Cycloheptane } & & & \\ \mathrm{H} & -1.186 & 2.319 & -0.522 \\ \mathrm{C} & -0.874 & 1.290 & -0.302 \\ \mathrm{C} & 0.147 & -0.749 & -1.535 \\ \mathrm{C} & 0.000 & -1.298 & 0.956 \\ \mathrm{C} & 0.874 & -1.290 & -0.302 \\ \mathrm{C} & 0.000 & 1.298 & 0.956 \\ \mathrm{C} & -0.147 & 0.749 & -1.535 \\ \mathrm{H} & -0.797 & -1.300 & -1.640 \\ \mathrm{H} & 0.314 & -2.113 & 1.619 \\ \mathrm{H} & 1.799 & -0.725 & -0.125 \\ \mathrm{H} & -0.314 & 2.113 & 1.619 \\ \mathrm{H} & 0.797 & 1.300 & -1.640 \\ \mathrm{H} & -1.799 & 0.725 & -0.125 \\ \mathrm{H} & 0.735 & -0.982 & -2.432 \\ \mathrm{H} & 0.875 & 0.011 & 2.430 \\ \mathrm{H} & 1.186 & -2.319 & -0.522 \\ \mathrm{C} & 0.000 & 0.000 & 1.769 \\ \mathrm{H} & -0.735 & 0.982 & -2.432 \\ \mathrm{H} & -0.875 & -0.011 & 2.430 \\ \mathrm{H} & -1.026 & -1.543 & 0.654 \\ \mathrm{H} & 1.026 & 1.543 & 0.654 \\ & & & \\ \mathrm{E}\left(\mathrm{QCISD} / 6-31 \mathrm{G}^{*}\right)=-172100.85 & \\ \mathrm{G} 3 \text { (MP2) Enthalpy=-172325.85 } & \end{array}$

$\begin{array}{lrrr}\text { Cyclopropene } & & & \\ \mathrm{H} & -0.913 & 1.456 & 0.000 \\ \mathrm{C} & 0.000 & 0.858 & 0.000 \\ \mathrm{C} & 0.000 & -0.499 & 0.651 \\ \mathrm{C} & 0.000 & -0.499 & -0.651 \\ \mathrm{H} & 0.913 & 1.456 & 0.000 \\ \mathrm{H} & 0.000 & -1.038 & -1.584 \\ \mathrm{H} & 0.000 & -1.038 & 1.584\end{array}$

$\mathrm{E}(\mathrm{QCISD} / 6-31 \mathrm{G} *)=-72947.08$ G3(MP2) Enthalpy=-73043.58

\section{Aza-phosphacyclobutane}

$\begin{array}{lrrr}\mathrm{H} & -1.422 & -0.176 & 1.294 \\ \mathrm{C} & -1.275 & -0.131 & 0.203 \\ \mathrm{~N} & -0.255 & -1.078 & -0.283 \\ \mathrm{C} & -0.451 & 1.116 & -0.158 \\ \mathrm{H} & -2.248 & -0.242 & -0.291 \\ \mathrm{H} & -0.538 & 1.977 & 0.506 \\ \mathrm{H} & -0.607 & 1.421 & -1.194 \\ \mathrm{P} & 1.074 & 0.037 & -0.010 \\ \mathrm{H} & 1.087 & 0.126 & 1.427 \\ \mathrm{H} & -0.242 & -2.015 & 0.119\end{array}$

$\mathrm{E}\left(\mathrm{QCISD} / 6-31 \mathrm{G}^{*}\right)=-298032.93$

G3(MP2) Enthalpy=-298207.60

\begin{tabular}{lrrr}
\multicolumn{4}{l}{ Aza-phosphacyclopentane } \\
$\mathrm{H}$ & -0.029 & 1.858 & \\
$\mathrm{C}$ & -0.040 & 1.361 & 0.031 \\
$\mathrm{P}$ & 1.294 & 0.028 & -0.044 \\
$\mathrm{~N}$ & 0.156 & -1.283 & -0.163 \\
$\mathrm{C}$ & -1.153 & -0.820 & 0.310 \\
$\mathrm{C}$ & -1.362 & 0.611 & -0.195 \\
$\mathrm{H}$ & 0.125 & 2.124 & -0.733 \\
$\mathrm{H}$ & -2.203 & 1.106 & 0.302 \\
$\mathrm{H}$ & 1.500 & -0.047 & 1.358 \\
$\mathrm{H}$ & -1.588 & 0.573 & -1.267 \\
$\mathrm{H}$ & 0.105 & -1.627 & -1.120 \\
$\mathrm{H}$ & -1.137 & -0.831 & 1.406 \\
$\mathrm{H}$ & -1.939 & -1.507 & -0.020
\end{tabular}

$\mathrm{E}\left(\mathrm{QCISD} / 6-31 \mathrm{G}^{*}\right)=-322638.46$

G3(MP2) Enthalpy=-322844.58 


$\begin{array}{lcrr}\text { Aza-phosphacyclohexane } & & \\ \mathrm{H} & 0.961 & 1.260 & 1.355 \\ \mathrm{C} & 1.013 & 1.247 & 0.259 \\ \mathrm{C} & 0.926 & -1.290 & 0.228 \\ \mathrm{P} & -1.505 & -0.023 & 0.090 \\ \mathrm{~N} & -0.412 & -1.273 & -0.358 \\ \mathrm{C} & -0.398 & 1.399 & -0.309 \\ \mathrm{C} & 1.696 & -0.044 & -0.192 \\ \mathrm{H} & 1.449 & -2.184 & -0.129 \\ \mathrm{H} & 1.790 & -0.049 & -1.284 \\ \mathrm{H} & -0.361 & 1.483 & -1.401 \\ \mathrm{H} & -0.869 & 2.314 & 0.068 \\ \mathrm{H} & 2.709 & -0.091 & 0.225 \\ \mathrm{H} & 1.627 & 2.107 & -0.036 \\ \mathrm{H} & 0.893 & -1.343 & 1.331 \\ \mathrm{H} & -1.307 & 0.068 & 1.510 \\ \mathrm{H} & -0.852 & -2.188 & -0.393 \\ \mathrm{E}(\mathrm{QCISD} / 6-31 \mathrm{G})=-371061.78 & \\ \mathrm{G} 3 \text { (MP2) Enthalpy=-371326.33 } & \\ \text { Aza-phosphacycloheptane } & & \\ \mathrm{H} & -0.376 & 1.277 & -1.464 \\ \mathrm{C} & -0.569 & 1.546 & -0.416 \\ \mathrm{C} & -1.777 & -0.752 & -0.120 \\ \mathrm{~N} & 0.649 & -1.336 & -0.338 \\ \mathrm{C} & -0.575 & -1.522 & 0.428 \\ \mathrm{C} & 0.702 & 1.384 & 0.426 \\ \mathrm{C} & -1.772 & 0.758 & 0.112 \\ \mathrm{H} & -2.691 & -1.163 & 0.329 \\ \mathrm{P} & 1.754 & -0.052 & -0.125 \\ \mathrm{H} & -0.813 & -2.594 & 0.445 \\ \mathrm{H} & 0.425 & 1.309 & 1.483 \\ \mathrm{H} & -2.681 & 1.167 & -0.346 \\ \mathrm{H} & -0.846 & 2.607 & -0.424 \\ \mathrm{H} & -1.847 & -0.958 & -1.197 \\ \mathrm{H} & 1.330 & 2.278 & 0.331 \\ \mathrm{H} & -1.372 & -1.232 & 1.466 \\ \mathrm{H} & -0.382 & 1.161 \\ \mathrm{H} & 0.954 & 1.189 \\ \mathrm{H}(\mathrm{QCISD} / 6-31 \mathrm{G} *)=-371811.17 & -1.309 \\ \mathrm{G} 3 \text { (MP2) Enthalpy=-372082.32 } & \\ & & & \\ & & \end{array}$

$\begin{array}{lrrr}\mathbf{N H} \mathbf{H}_{2}-\mathbf{C}\left(\mathbf{P H}_{\mathbf{2}}\right)=\mathbf{C H}_{\mathbf{2}} & & \\ \mathrm{N} & 1.119 & -1.177 & 0.090 \\ \mathrm{C} & 0.487 & 0.076 & -0.001 \\ \mathrm{C} & 1.151 & 1.245 & -0.001 \\ \mathrm{P} & -1.344 & -0.075 & -0.088 \\ \mathrm{H} & 2.238 & 1.275 & -0.026 \\ \mathrm{H} & 0.633 & 2.193 & 0.050 \\ \mathrm{H} & 0.738 & -1.872 & -0.546 \\ \mathrm{H} & 2.128 & -1.119 & -0.023 \\ \mathrm{H} & -1.593 & -0.345 & 1.279 \\ \mathrm{H} & -1.639 & 1.305 & -0.027 \\ \mathrm{E}\left(\mathrm{QCISD} / 6-31 \mathrm{G}^{*}\right)=-298029.09 & \\ \mathrm{G} 3(\mathrm{MP} 2) \text { Enthalpy=-298207.52 } & \end{array}$

$\begin{array}{lrrr}\mathbf{M e}_{2}-\mathbf{C}=\mathbf{C H}_{2} & & & \\ \mathrm{H} & 0.000 & 0.924 & 2.028 \\ \mathrm{C} & 0.000 & 0.000 & 1.459 \\ \mathrm{C} & 0.000 & 0.000 & 0.120 \\ \mathrm{H} & 0.000 & -0.924 & 2.028 \\ \mathrm{C} & 0.000 & 1.273 & -0.679 \\ \mathrm{C} & 0.000 & -1.273 & -0.679 \\ \mathrm{H} & 0.880 & 1.324 & -1.330 \\ \mathrm{H} & 0.000 & 2.153 & -0.032 \\ \mathrm{H} & -0.880 & 1.324 & -1.330 \\ \mathrm{H} & -0.880 & -1.324 & -1.330 \\ \mathrm{H} & 0.000 & -2.153 & -0.032 \\ \mathrm{H} & 0.880 & -1.324 & -1.330 \\ \mathrm{E}\left(\mathrm{QCISD} / 6-31 \mathrm{G}^{*}\right)=-98327.77 & \\ \mathrm{G} 3(\mathrm{MP} 2) \text { Enthalpy=-98460.19 } & \end{array}$

$\begin{array}{lrrr}\mathbf{N H}_{2}-\mathbf{C H}\left(\mathbf{P H}_{2}\right)-\mathbf{C H}_{3} & & \\ \mathrm{H} & -0.493 & 0.087 & -1.493 \\ \mathrm{C} & -0.436 & 0.059 & -0.399 \\ \mathrm{P} & 1.390 & -0.168 & 0.002 \\ \mathrm{C} & -1.206 & -1.161 & 0.092 \\ \mathrm{~N} & -1.101 & 1.264 & 0.094 \\ \mathrm{H} & -0.807 & -2.086 & -0.332 \\ \mathrm{H} & -2.257 & -1.063 & -0.188 \\ \mathrm{H} & -1.152 & -1.238 & 1.184 \\ \mathrm{H} & -1.036 & 1.289 & 1.112 \\ \mathrm{H} & -0.610 & 2.094 & -0.238 \\ \mathrm{H} & 1.801 & 1.158 & -0.289 \\ \mathrm{H} & 1.266 & 0.045 & 1.400 \\ \mathrm{E}\left(\mathrm{QCISD} / 6-31 \mathrm{G}^{*}\right)=-298782.72 & \\ \mathrm{G} 3(\mathrm{MP} 2) \text { Enthalpy=-298963.01 } & \end{array}$




$\begin{array}{lrrr}\text { NH}_{2} \text {-PH-CH3 } & & & \\ \mathrm{H} & -2.200 & 0.254 & -0.373 \\ \mathrm{~N} & -1.300 & 0.582 & -0.044 \\ \mathrm{H} & -1.409 & 1.130 & 0.804 \\ \mathrm{P} & -0.066 & -0.619 & -0.085 \\ \mathrm{C} & 1.416 & 0.471 & -0.014 \\ \mathrm{H} & 0.015 & -1.071 & 1.266 \\ \mathrm{H} & 2.310 & -0.136 & 0.152 \\ \mathrm{H} & 1.353 & 1.219 & 0.781 \\ \mathrm{H} & 1.523 & 0.988 & -0.969 \\ \mathrm{E}\left(\mathrm{QCISD} / 6-31 \mathrm{G}^{*}\right)=-274207.16 & \\ \mathrm{G} 3 \text { (MP2) Enthalpy=-274345.08 } & \end{array}$

\section{$\mathrm{PH} 2-\mathrm{C}\left(\mathrm{CH}_{3}\right)=\mathrm{CH}_{2}$}

$\begin{array}{lrrr}\mathrm{H} & 1.518 & -0.914 & 1.037 \\ \mathrm{P} & 1.373 & 0.045 & 0.000 \\ \mathrm{H} & 1.518 & -0.914 & -1.037 \\ \mathrm{C} & -0.463 & 0.122 & 0.000 \\ \mathrm{C} & -1.036 & 1.336 & 0.000 \\ \mathrm{C} & -1.274 & -1.143 & 0.000 \\ \mathrm{H} & -2.346 & -0.926 & 0.000 \\ \mathrm{H} & -1.045 & -1.753 & 0.880 \\ \mathrm{H} & -1.045 & -1.753 & -0.880 \\ \mathrm{H} & -0.447 & 2.248 & 0.000 \\ \mathrm{H} & -2.117 & 1.452 & 0.000\end{array}$

$\mathrm{E}\left(\mathrm{QCISD} / 6-31 \mathrm{G}^{*}\right)=-287979.85$

G3(MP2) Enthalpy=-288140.22

\section{Ethane}

$\begin{array}{lrrr}\mathrm{H} & 0.000 & 1.019 & 1.157 \\ \mathrm{C} & 0.000 & 0.000 & 0.762 \\ \mathrm{H} & 0.882 & -0.509 & 1.157 \\ \mathrm{C} & 0.000 & 0.000 & -0.762 \\ \mathrm{H} & -0.882 & -0.509 & 1.157 \\ \mathrm{H} & 0.882 & 0.509 & -1.157 \\ \mathrm{H} & -0.882 & 0.509 & -1.157 \\ \mathrm{H} & 0.000 & -1.019 & -1.157 \\ \mathrm{E}\left(\mathrm{QCISD} / 6-31 \mathrm{G}^{*}\right)=-49908.68 & \\ \text { G3(MP2) Enthalpy }=-49979.06 & \end{array}$

\section{Ethene}

$\begin{array}{lrrr}\mathrm{H} & 0.923 & 0.000 & -1.238 \\ \mathrm{C} & 0.000 & 0.000 & -0.667 \\ \mathrm{C} & 0.000 & 0.000 & 0.667 \\ \mathrm{H} & -0.923 & 0.000 & -1.238 \\ \mathrm{H} & 0.923 & 0.000 & 1.238 \\ \mathrm{H} & -0.923 & 0.000 & 1.238\end{array}$

$\mathrm{E}\left(\mathrm{QCISD} / 6-31 \mathrm{G}^{*}\right)=-49147.87$

G3(MP2) Enthalpy=-49216. 